\title{
Lung Function in Type 1 and Type 2 Diabetes Mellitus
}

\author{
Dr. Jagadesha CG ${ }^{1}$, Dr. Srinivas Raju D. $*^{2}$ \\ ${ }^{1}$ Assistant Professor, Department of General Medicine, Adichunchangiri Institute of Medical Sciences, B.G Nagar, Mandya \\ ${ }^{2}$ Assistant Professor, Department of Neurology, Vydehi Institute of Medical Science, Whitefield, Bangalore \\ *Corresponding Author: Dr. Srinivas Raju D.; abstractsubmissionnow@gmail.com
}

Received 21 June 2019;

Accepted 06 July 2019;

Published 15 July 2019

\begin{abstract}
Current estimates indicate that $8.3 \%$ of the adult population, or 71.4 million people, have diabetes in $2011,61.3$ million of whom are in India. The incidence rate for type 1 diabetes in India was frequently used in extrapolation for other countries in the region and therefore plays a pivotal role in the estimates. We evaluated the ventilatory function of type 1 diabetes mellitus and type 2 diabetes mellitus patients. Spirometry and DLCO conducted in patients who have never smoked, with no history of lower respiratory illness and at examination did not have any respiratory symptoms like nasal itching, nasal congestion, running nose, dry throat, hoarseness, epistaxis, sneezing, pain suggestive of sinusitis, cough, expectoration and dyspnea were included. DLCO $<80 \%$ is statistically similar in two groups with $\mathrm{P}=0.431$. Mean FEV1/FVC (measured $\%$ ) according to Diabetic duration, diabetic treatment, diabetic complications and DLCO in Type II DM patents was significant. Spirometric values were consistently lower in subjects with Type 1 diabetes mellitus than in Type 2 diabetics. The effect on the FVC was even more pronounced in diabetics who had duration of disease longer than 5 years, and the effect was not explained by the difference in age alone. Subjects with poorer diabetic control have worse spirometric function. Non-enzymatic glycosylation of connective tissue, especially the collagen, may be responsible for reduced lung functions.
\end{abstract}

\section{Introduction}

Current estimates indicate that $8.3 \%$ of the adult population, or 71.4 million people, have diabetes in $2011,61.3$ million of whom are in India.

The number of people with diabetes in the region will increase to 120.9 million by 2030 , or $10.2 \%$ of the adult population. A further 23.8 million people have impaired glucose tolerance (IGT) in 2011, and this will increase to 38.6 million by 2030. The SouthEast Asia Region has one of the highest estimates of prevalence of type 1 diabetes in children, with 111,500 affected. In 2011, an estimated 18,000 children under the age of 15 in the region developed type 1 diabetes. India accounts for most of the children with type 1 diabetes in the region. The incidence rate for type 1 diabetes in India was frequently used in extrapolation for other countries in the region and therefore plays a pivotal role in the estimates. This region contributes more than any other to the worldwide total. ${ }^{[1]}$ WHO projects that diabetes deaths will increase by two thirds between 2008 and 2030. ${ }^{[2]}$

\section{Objective}

To study the ventilatory function of type 1 diabetes mellitus and type 2 diabetes mellitus patients.

\section{Methods}

Patients with diabetes mellitus, both newly diagnosed as well as known cases admitted in medical wards or attending the outpatient department at a tertiary care teaching hospital. 80 diabetic patients of the age 18 years and above, 30 type 1 diabetes and 50 individuals who are type 2 diabetes mellitus randomised with detailed medical examination. Spirometry and DLCO conducted with a prior consent of the patient.

\section{Inclusion criteria}

Diabetics who have never smoked, with no history of lower respiratory illness and at examination did not have any respiratory symptoms like nasal itching, nasal congestion, running nose, dry throat, hoarseness, epistaxis, sneezing, pain suggestive of sinusitis, cough, expectoration and dyspnea were included.

\section{Exclusion criteria}

Patients who were smokers, present or past history of respiratory diseases that might affect lung function such as asthma, COPD, tuberculosis, bronchiectasis, interstitial lung disease, history of occupational exposure to any substances that could affect lung function, individuals with current or recent upper respiratory or lower respiratory infection, that could pre-dispose to heightened airway reactivity, individuals with unacceptable spirometric 
technique (in which FEV1 or FVC could not be correctly measured due to cough, obstruction of teeth or tongue, sub-maximal effort for air escape, effort sustained for less than 6 seconds duration of

failure to attain a plateau on volume time curve, lack of understanding of the procedures, recent surgery)

\section{Results}

Table 1: Distribution of treatment in two groups of patients studied

\begin{tabular}{|l|c|c|c|c|}
\hline \multirow{2}{*}{ Treatment } & \multicolumn{2}{|c|}{ Type I DM } & \multicolumn{2}{c|}{ Type II DM } \\
\cline { 2 - 5 } & No & \% & No & \% \\
\hline Insulin & 30 & 100.0 & 0 & 0.0 \\
OHA & 0 & 0.0 & 39 & 78.0 \\
OHA \& INS & 0 & 0.0 & 11 & 22.0 \\
Total & 30 & 100.0 & 50 & 100.0 \\
\hline
\end{tabular}

Table 2: Distribution of DLCO in two groups of patients studied

\begin{tabular}{|l|c|c|c|c|}
\hline \multirow{2}{*}{ DLCO } & \multicolumn{2}{|c|}{ Type I DM } & \multicolumn{2}{c|}{ Type II DM } \\
\cline { 2 - 5 } & No & \% & No & \% \\
\hline$<80.0 \%$ & 8 & 61.5 & 5 & 45.5 \\
$>80.0 \%$ & 5 & 38.5 & 6 & 54.5 \\
Total & 13 & 100.0 & 11 & 100.0 \\
Not recorded & 17 & - & 39 & - \\
\hline
\end{tabular}

DLCO $<80 \%$ is statistically similar in two groups with $P=0.431$

Table 3: Mean FEV1/FVC (measured \%) according to Diabetic duration, diabetic treatment, diabetic complications and DLCO in Type I DM patents

\begin{tabular}{|c|c|c|c|c|}
\hline \multirow[b]{2}{*}{ Variables } & \multicolumn{3}{|c|}{ FEV1/FVC (measured \%) in Type I DM } & \multirow[b]{2}{*}{$P$ value } \\
\hline & Min-Max & Mean \pm SD & $95 \% \mathrm{CI}$ & \\
\hline \multicolumn{5}{|c|}{ Diabetic Duration in months } \\
\hline $\begin{array}{ll} & <24 \\
\text { - } & 25-60 \\
\text { - } & 61-120 \\
\text { - } & >120\end{array}$ & $\begin{array}{c}88.04-90 \\
73.49-87.19 \\
60.23-87.29 \\
29.5-94.9\end{array}$ & $\begin{array}{c}89.02 \pm 1.39 \\
81.75 \pm 5.83 \\
73.51 \pm 11.05 \\
71.8 \pm 16.28\end{array}$ & $\begin{array}{c}76.57-101.47 \\
72.48-91.02 \\
55.92-91.1 \\
64.18-79.42\end{array}$ & $\begin{array}{l}\mathrm{F}=1.230 \\
\mathrm{P}=0.319\end{array}$ \\
\hline \multicolumn{5}{|l|}{ Diabetic treatment } \\
\hline $\begin{array}{ll}- & \text { Insulin } \\
\text { - } & \text { OHA } \\
\text { - } & \text { OHA \& INS }\end{array}$ & $\begin{array}{c}29.5-94.9 \\
- \\
-\end{array}$ & $\begin{array}{c}74.5 \pm 14.73 \\
- \\
-\end{array}$ & $\begin{array}{c}69-80 \\
- \\
-\end{array}$ & $\begin{array}{l}- \\
- \\
-\end{array}$ \\
\hline \multicolumn{5}{|l|}{ Diabetic complications } \\
\hline $\begin{array}{ll}- & \text { No } \\
\text { - } & \text { Yes }\end{array}$ & $\begin{array}{c}52-94.9 \\
29.5-86.09\end{array}$ & $\begin{array}{l}78.35 \pm 10.83 \\
65.52 \pm 19.05\end{array}$ & $\begin{array}{l}73.42-83.28 \\
50.88-80.17\end{array}$ & $\begin{array}{l}\mathrm{t}=2.3515 \\
\mathrm{p}=0.026^{*}\end{array}$ \\
\hline \multicolumn{5}{|l|}{ DLCO } \\
\hline $\begin{array}{ll}- & <80.0 \% \\
\bullet & >80.0 \%\end{array}$ & $\begin{array}{l}29.5-73 \\
68.1-74\end{array}$ & $\begin{array}{c}57.49 \pm 14.83 \\
72.52 \pm 2.48\end{array}$ & $\begin{array}{c}45.09-69.89 \\
69.44-75.6\end{array}$ & $\begin{array}{c}\mathrm{t}=2.210 \\
\mathrm{p}=0.049 *\end{array}$ \\
\hline
\end{tabular}

Table 4: Mean FEV1/FVC (measured \%) according to Diabetic duration, diabetic treatment, diabetic complications and DLCO in Type II DM patents

\begin{tabular}{|c|c|c|c|c|}
\hline \multirow[b]{2}{*}{ Variables } & \multicolumn{3}{|c|}{ FEV1/FVC (measured \%) in Type II DM } & \multirow[b]{2}{*}{ P value } \\
\hline & Min-Max & Mean \pm SD & $95 \% \mathrm{CI}$ & \\
\hline \multicolumn{5}{|c|}{ Diabetic Duration in months } \\
\hline$\bullet \quad<24$ & $81.49-96.79$ & $89.29 \pm 4.53$ & $85.81-92.77$ & \\
\hline - $\quad 25-60$ & $60.85-93.07$ & $80.94 \pm 11.29$ & $73.36-88.53$ & $\mathrm{~F}=3.017$ \\
\hline - $61-120$ & $60.85-99.71$ & $78.26 \pm 9.69$ & $72.9-83.63$ & $\mathrm{P}=0.039^{*}$ \\
\hline - $\quad>120$ & $65.41-95.92$ & $79.03 \pm 9.48$ & $73.78-84.28$ & \\
\hline \multicolumn{5}{|l|}{ Diabetic treatment } \\
\hline$\bullet \quad$ Insulin & - & - & - & \\
\hline - $\mathrm{OHA}$ & $60.85-99.71$ & $81.29 \pm 10.08$ & $78.02-84.56$ & $\mathrm{t}=0.293$ \\
\hline - $\quad$ OHA \& INS & $62.32-92.05$ & $80.28 \pm 9.64$ & $73.8-86.75$ & $\mathrm{p}=0.768$ \\
\hline \multicolumn{5}{|l|}{ Diabetic complications } \\
\hline$\bullet \quad \mathrm{No}$ & $60.85-96.79$ & $84.03 \pm 9.04$ & $80.38-87.69$ & $\mathrm{t}=2.302$ \\
\hline - $\quad$ Yes & $60.85-99.71$ & $77.85 \pm 9.95$ & $73.65-82.05$ & $\mathrm{p}=0.026^{*}$ \\
\hline \multicolumn{5}{|l|}{ DLCO } \\
\hline$\bullet \quad<80.0 \%$ & $65.41-74.16$ & $71.28 \pm 3.53$ & $66.9-75.67$ & $\mathrm{t}=1.934$ \\
\hline - $\quad>80.0 \%$ & $60.85-71.44$ & $66 \pm 5.16$ & $60.59-71.42$ & $\mathrm{p}=0.085+$ \\
\hline
\end{tabular}




\section{Discussion}

Marco Guazzi ${ }^{[4]}$ and colleagues in their in his study in chronic congestive heart failure (CHF) states that the backward effects of left ventricular dysfunction alters pulmonary volumes and gas diffusion. They explored the possibility that coexistence of noninsulin-dependent diabetes mellitus (NIDDM) may potentiate the deterioration of lung function in CHF.

In 20 normoglycemic patients (group 1) and in 20 patients with NIDDM (group 2), with New York Heart Association class II to class III CHF due to idiopathic or ischemic cardiac disease, and in 20 controls (groups were age - and gender-matched ), they investigated the following:

- Cardiac function

- Pulmonary volumes

- Carbon monoxide diffusion (DLCO) and its alveolar capillary membrane (DM) subcomponent

- $\quad$ Oxygen uptake and dead space-to-tidal volume ratio ( $\mathrm{p}$ $\mathrm{VD} / \mathrm{VT}$ ) at peak exercise (individualized ramp test)

- Slope of ventilation-to-carbon dioxide production ratio (VE/VCO2) during exercise.

Although, compared with reference subjects, both patient groups had similar variations in left ventricular diastolic volume, ejection, $\&$ pulmonary wedge pressure, in group 2 lung volumes, DLCO, $\mathrm{DM}$, and oxygen uptake were significantly more reduced; in this group there was no overlap of individual results of DLCO and DM with those in controls; VE/VCO2 Slope \& $\mathrm{p}$ VD/VT also were significantly increased and inversely correlated with DM. Thus, coexistence of NIDDM makes pulmonary dysfunction worse in $\mathrm{CHF}$, and significantly exercise intolerance. Because of the integrated function of heart and lungs, it is difficult for one component to be compromised without altering the physiology of the other. Cardiac disease can lead - mainly through the effects of pulmonary venous hypertension - to a restrictive or obstructive respiratory pattern \& impeded gas transfer. DM increases the likelihood of development of congestive cardiac failure from all causes, \& even when patients with prior coronary or rheumatic heart disease are excluded, diabetic subjects have a four to fivefold increased risk of CHF, suggesting that the probability of developing respiratory dysfunction may be similarly raised. In addition, the presence in the lung of an extensive microvascular circulation \& plentiful connective tissue raises the possibilities that in DM, microvascular circulation and nonenzymatic glycosylation of tissue proteins thicken the alveolar epithelial and alter lung mechanics and gas exchange independently of myocardial dysfunction. Sixty percent of persons with adult DM have abnormal pulmonary function. Impairment in gas transfer has also been documented in several adult patients with insulin -dependent diabetes mellitus. Hiroshi Mori et al, ${ }^{[5]}$ examined the possible association between the vascular complications of diabetes \& changes in pulmonary function, they performed pulmonary functions tests including assessment of the diffusing capacity (\%DLCO) in 80 patients with non-insulin-dependent diabetes mellitus (45 males and 35 females) without overt lung or heart disease was performed. The mean age of the subjects was 57.9 years and the mean duration of diabetes was 10.8years. The \% DLCO decreased significantly as the duration of diabetes increased $(\mathrm{r}=-0.38, \mathrm{p}$ less than 0.01 ), and the same relationship was also observed in non-smoking individuals $(\mathrm{n}=37)$. The reduction in $\%$ DLCO was greater in patients with diabetic microangiopathy (especially nephropathy) and in those treated with insulin. Other spirometric functions (\%VC,FEV1.0,PaO2 and $\mathrm{PaCO} 2$ ) showed no relationship to the duration of DM, the degree of microangiopathy or the type of treatment. \%DLCO was found to correlate negatively with duration of diabetes. Asanuma et al, ${ }^{[8]}$ and Sandler et al, ${ }^{[32]}$ have reported that as the duration of diabetes is increased, the pulmonary diffusion capacity is decreased.

Uchida et al, ${ }^{[6]}$ in a study of ventilation-perfusion scintigrams in diabetics, observed a decreased pulmonary diffusing capacity in patients with perfusion defect. As these patients had a longer duration of diabetes and a higher incidence of retinopathy, pulmonary microangiopathy was suggested. P. Lang et al, ${ }^{[7]}$ studied possible associations between DM, plasma glucose, forced vital capacity (FVC) and forced expiratory volumes in one second (FEV1). They analyzed the results from a cross sectional study of 11,763 subjects. The subjects were 20 years or older and were representative of the population of Copenhagen city. 284 of participants had DM as assessed by questionnaire. On average, FVC was reduced by $334 \mathrm{ml}$, and FEV1 by $239 \mathrm{ml}$ in diabetic subjects treated with insulin, and by $184 \mathrm{ml}$ and $117 \mathrm{ml}$ respectively in diabetic subjects treated with hypoglycemic agents and /or diet compared to control subjects.

Their results show that in a population study both IDDM and NIDDM are associated with slightly reduced values of FEV1 and FVC. The reduction of lung function is slightly more pronounced in diabetic subjects without treatment. This suggests that the severity of DM may influence the degree of lung function impairment. The highly significant association between raised values of plasma glucose and impairment of lung function substantiates this possibility.

Previous spirometric studies on subjects with Diabetes mellitus have been conducted on highly selected patients with IDDM. Although the majority of the investigators have reported changes in the elastic properties of the lung and reduced pulmonary diffusing capacity, ${ }^{[27,32,35,36]}$ FEV1 and FVC have mostly been within normal range. ${ }^{[27,32,35,36]}$

However, in the study of ANASUMA et al, ${ }^{[8]}$ the subjects with IDDM had slightly but significantly reduced FEV1 and FVC compared to control subjects.

In another study, Schnapf et al, ${ }^{[9]}$ found that there was reduction of lung volumes in IDDM patients when the patients also had decreased joint mobility. Consequently, it has been suggested that non-enzymatic glycosylation of connective tissue, especially the collagen, might be responsible for both lung and joint abnormalities. Their findings of an association between raised values of plasma glucose and lung function impairment are in accordance with this hypothesis.

Due to the small number of diabetic subjects in many of the subgroups, the study does not allow detailed analysis of the impact of DM on ventilatory function in the different age groups. Even so, the slight lung function reduction in the diabetic subjects was present in all age groups and there was no significant interaction between age and DM in the regression analysis. Many confounding factors might lead to reduction of both FEV1 and FVC in diabetic subjects. Two of them are obesity and cardiac failure. As many subjects with NIDDM are obese, the reduction of FEV1 and FVC in NIDDM might therefore be the result of being overweight rather than the result of NIDDM. However, since they included BMI in 
the regression model, obesity was unlikely to be an explanation for observed lung function impairment. In addition, the most pronounced lung function impairment was in the DM 1 group. Although none of the diabetic subjects had manifest heart failure during the examination, it was not possible to exclude that a mild pulmonary congestion was present in some of the diabetic subjects, as ischemic heart disease is more prevalent in subjects with DM than in normals.

G. Engstorm et al, ${ }^{[10]}$ investigated whether reduced lung function is a risk factor for developing diabetics. They studied non-diabetic men $(n=382)$ from a population- based cohort - "men born in 1914 "- who were examined with spirometry at age 55years. The cohort was re-examined at age 68years. Diabetics fasting plasma glucose at follow -up were studied in relation to vital capacity (VC) and forced expiratory volume (FEV1) at base line. Their results showed 15 men developed diabetes during follow up. The percentage with diabetes during in the 1st,2nd,3rd and top quartile of vital capacity were $7 \%, 5 \%, 2 \%$, and $1 \%$, respectively (p for trend $=0.01)$. Fasting glucose $(\log$ transformed, mmol/l) at follow up was $1.63+/-0.16,1.62+/-0.18,1.61+/-0.11$ and $1.60+/-0.11$, respectively $(\mathrm{P}$ for trend $=0.11$ ). The longitudinal associations between VC and diabetics $(\mathrm{P}=0.001)$ and log glucose $(\mathrm{P}=0.036)$ were significant after adjustment for several potential confounders. FEV1.0 at base line showed similar associations with diabetes at follow up. A second limitation is the small number that developed diabetes.

The casual associations between lung function and future diabetes remain to be evaluated. Inflammatory processes often play key roles in the development of reduced lung function. There is also a growing recognition of association between the immune system and metabolism of glucose and insulin. It is possible that susceptible individuals have a greater tendency to react with inflammation, which could cause reduced lung function, insulin resistance and type 2 diabetes. Another possibility is that reduced lung function and risk of diabetes are both partially determined by early growth. Low birth weight has been associated both with type 2 diabetes and reduced adult lung function.

The conclusion of this population-based study of middle aged men is that lung function is universally associated with the risk of future diabetes.

Marko Guzzi et al also in a later study showed that, insulin improves alveolar-capillary membrane gas conductance in type 2 DM They studied 19 life-long non-smoking, asymptomatic patients with type $2 \mathrm{DM} \&$ normal cardiac function, whose HbA1c averaged $6.2+/-0.3 \%$ with diet \& hypoglycemic drugs. DLCO \& its subcomponents (alveolar capillary membrane conductance Dm \& pulmonary capillary blood volume available for gas exchange Vc, forced expiratory volume in 1s FEV1, cardiac output $\mathrm{CO}$, ejection fraction $\mathrm{EF}$, pulmonary wedge pressure WPP, and pulmonary arteriolar resistance PAR were determined before \& within $60 \mathrm{~min}$ after infusion of $50 \mathrm{ml}$ saline $+10 \mathrm{IU}$ of regular insulin or after saline alone on 2 consecutive days (random block design). Glycemia was kept at base line levels during experiments by dextrose infusion.

Results showed percent of normal predicted DLco averaged 84.2 $+/-7.9 \%$ \& in 14 patients was $<100 \%$. Insulin infusion, not saline alone, improved (P < 0.01) DLCO (12\%) and DM (14\%) \& raised DLco to $98 \%$ of the normal predicted value. There were no variations in $\mathrm{VC}, \mathrm{FEV} 1, \mathrm{CO}, \mathrm{EF}$, WPP, or PAR, suggesting that the influences of the hormone on gas transfer was not mediated by changes in spirometry, volumes, and hemodynamics of the lung.

Spomenka Ljubic et $\mathrm{al}^{[11]}$ study showed a reduction of diffusion capacity for carbon monoxide in DM patients. Diabetes can cause the development of pulmonary complications due to collagen \& elastin changes, as well as microangiopathy. This study demonstrates the relationship between pulmonary complications \& other chronic complications in diabetes. 27 patients with diabetes, aged 21 to $62 \mathrm{yrs}$, who had the disease from 3 to $32 \mathrm{yrs,}$, were included in this study.The protein excretion rate (PER) and the diffusion capacity of the lung for carbon monoxide (DLCO) were included as parameters of the severity of complications. PER was determined by Biuret method. DLCO was measured by single breath method \& was corrected by the measurement of alveolar volume (VA). The values of DLco as corrected by VA (DLCO/VA) were included in the statistical evaluation of the results. The variations of age, duration of DM, and complication parameters were included in a multiple regression model with forward, stepwise selection to asses their value in predicting DLco /VA. The variables were found to be significant predictors of DLco /VA $(\mathrm{R} 2=0.46, \mathrm{R} 2=0.32, \mathrm{p}<0.022)$. However, proteinuria was the only significant independent predictor of DLCO /VA. This finding indicates that both renal and pulmonary complications of diabetes share a similar microangiopathic background.

SK Rajan et $\mathrm{al}^{[12]}$ study of spirometric evaluation of type $1 \mathrm{DM}$, was a cross sectional study of 30 patients (group 1) who are on insulin therapy. Group 2 consisted of age matched, sex matched, non-smoking healthy volunteers who acted as controls. Spirometric revealed normal findings in 10 patients (33\%), \& abnormal findings in 20 patients $(67 \%)$. Among these 20 patients $(67 \%)$ with abnormal findings, obstructive pattern was present in 18 patients $(60 \%)$, restrictive pattern was present in 9 patients $(30 \%)$ and mixed pattern was observed in 7 patients (23\%). This study showed that the lung has to be considered as one of the target organs in type $1 \mathrm{DM}$. In view of the possibility pneumopathy in asymptomatic type1DM, all persons with long - standing type 1DDM should undergo regular assessment of pulmonary function.

DM is associated with widespread hormonal, metabolic, and micro vascular abnormality, as well as with disturbances of the function of many organ systems. The kidneys, eyes, cardio vascular system, and respiratory system can be damaged. The aim of this study was to investigate lung function in DM and renal complications. The development of these complications could be explained by the biochemical alteration of connective tissue constituents, particularly collagen and elastin, as well as micro angiopathy due to a non enzymatic glycosylation of proteins induced by chronic hyperglycemia. Collagen is an abundant structural protein found in the previously mentioned organic systems, so the disturbance of the function of those systems can be expected. The kidneys, eyes and lungs of patients with diabetes are affected consequently, and the patient develops obstructive and restrictive disorders. As a result of alveolar-capillary membrane thickenings due to collagen and elastin alterations and microangiopathy, the capacity for the diffusion of carbon monoxide is reduced.

The mean HbA1c levels in type 1 diabetes was $6.79 \pm 0.55$ and type 2 diabetes was $6.97 \pm 0.68$ which all showed that type 2 diabetes subjects had a poor glycemic control compared to type 1 diabetes mellitus subjects. FEV1/FVC (measured \%) of Type $1 \mathrm{DM}$ is $74.50 \pm 14.73$ which is significantly low. Asanuma et al study on type $1 \mathrm{DM}$ also showed reduced FEV1/FVC \& 90\& P.Lang et al 
study on DM also showed reduced FEV1/FVC similar to our study. FEV1/FVC (measured \%) of Type $2 \mathrm{DM}$ is $81.07 \pm 9$. Diffusion capacity of both groups had similar results i.e DLCO $<80 \%$ is statistically similar in two groups with $\mathrm{P}=0.431$.In Marico Guzzi et all study the mean DLCO was $84.2+/-7.9 \%$. The studies conducted have mainly focused on alterations in diffusing capacity (DLCO) and their relationship with duration of diabetes, in insulin dependent diabetes mellitus. They found that there was reduction in lung function that was slightly more pronounced in insulin dependent than in non-insulin dependent diabetics. we also noted the same pattern in our study.

The groups were also homogeneous in respect of having no known respiratory disease, and all being non-smokers. In the study of Hiroshi Mori et al, smokers were included in the analysis, and this was therefore an additional confounding variable.

\section{Conclusions}

Spirometric values were consistently lower in subjects with Type 1 diabetes mellitus than in Type 2 diabetics. The Age distribution was Mean \pm SD $31.67 \pm 6.41 \&$ Mean \pm SD 54.48 \pm 8.83in type $1 \&$ 2 diabetics respectively. BMI of type 1 diabetic showed Mean \pm SD 21.04 \pm 2.00 and type 2 diabetic showed Mean \pm SD 27.03 \pm 2.46 . Duration of DM Duration of disease is significantly less in Type 2 DM compared to Type 1DM with Diabetic complications were seen in both the groups and nephropathy was the common complication followed by non proliferative diabetic retinopathy and neuropathy. Presence of complications more in Type 2 DM compared to Type 1 DM. The mean fasting glucose levels in type 1 diabetes was $158.80 \pm 53.10 \mathrm{mg} / \mathrm{dl} \&$ type 2 diabetes was $169.72 \pm 54.06 \mathrm{mg} / \mathrm{dl}$. The mean post prandial glucose levels in type 1 diabetes was $213.00 \pm 61.84 \mathrm{mg} / \mathrm{dl} \&$ type 2 diabetes was $225.76 \pm 70.97 \mathrm{mg} / \mathrm{dl}$ The mean HbA1c levels in type 1 diabetes was $6.79 \pm 0.55$ and type 2 diabetes was $6.97 \pm 0.68$. Type 2 diabetes subjects had a poor glycemic control compared to type 1 diabetes mellitus subjects. FEV1/FVC (measured \%) of Type $1 \mathrm{DM}$ is $74.50 \pm 14.73$ compared to FEV1/FVC (measured \%) of Type $2 \mathrm{DM}$ is $81.07 \pm 9$. Diffusion capacity of both groups had similar results i.e DLCO $<80 \%$. The effect on the FVC was even more pronounced in diabetics who had duration of disease longer than 5 years, and the effect was not explained by the difference in age alone. Subjects with poorer diabetic control have worse spirometric function. Non-enzymatic glycosylation of connective tissue, especially the collagen, may be responsible for reduced lung functions. There is scope for further intensive work in the same area, extending the study to a larger group \& multicentric study to work on ethnicity, geography, culture and more.

\section{Bibliography}

[1] International Diabetic Federation- South East Asia journal, Jan2012.

[2] WHO-Geneva conclave 2012.

[3] The Diabetic Epidemic of India- Safia Fatima mohiuddin jan 21,2012.

[4] Marco Guazzi MD, Gianluco Pantane,MD, Piegiuseppe Agastani, MD, PhD, and Maurizio D. Guazzi MD, PhD Effect of NIDDM on pulmonary function and Exercise Tolerance in chronic congestive heart failure. Amer Jour Card Vol 89. Jan2002

[5] Hiroshi Mori, Masamichi Okuba,Midori Qkamura, Kiminori Yamane,Seijiro Kado, Genshi Egusa, Takehiko
Hiramoto, Hitoshi \& Michi Yamakido. Abnormalities of pulmonary function in patients with NIDDM Internal Medicine 31, 2, 189-193.

[6] Uchida $\mathrm{K}$ et al The findings of ventilation \& perfusion scintigrams in patients with DM Resiper res 7: 345, 1988

[7] P. Lange, S.Groth, J. Kastrup, M. Appleyard, J. Jansen, P. Schnohr Diabetes Mellitus, plasma glucose \& lung function in a cross-sectional population study Eur Resp J 1989, 2.14-19.

[8] Asanuma $\mathrm{Y}$ et al Characteristics of pulmonary function in patients with DM Diabetes Res clin pract I; 95.1985.

[9] Schnaph BM et al. Pulmonary function in IDDM with limited joint mobility. Am Rev Respir Dis 130: 930.1984.

[10] G. Engstrom \& L. Janzon Risk of developing diabetes is inversely related to lung function: a population based cohort study Diabetes Med 2002, 19, 167-170.

[11] Spomenka Ljubic, MD, Msc; Zeljko Metelko, MD, PhD; Nikica Car, MD, PhD; Gojka Roglic, MD; and Zinka drazic, MD Reduction of Diffusion Capacity for Carbon Monoxide in diabetic patients CHEST/ 114/4/OCT 1998.

[12] SK Rajan, P Rajesh Prabhu, Madhu Sasidharan, Spirometric evaluation of type1 DM Abstract of paper presented at APICON 2003, JAPI Vol 50, 1529 December 2002

[13] Ozmen B et al Pulmonary function parameters in patients with DM. Diabetes Research \& Clinical Practice 57 (2002) 209 - 211.

[14] Marco Guazzi, MD, PhD. Jacopo Oeglia, MD. Maurizio D. Guazzi, MD, PhD. Insulin improves Alveolarcapillary Membrane Gas conductance in Type 2 DM Diabetes Care 25(2002)1802-1812.

[15] Naam C et al. Pulmonary function abnormalities in chronic severe cardiomyopathy preceding cardiac transplantations Am Rev Resp Dis 1992; 145:1334-1338.

[16] Guazzi et al. Improvement in alveolar-capillary membrane diffusing capacity with enalapril in CCF \& counteracting effect of aspirin: Circulation 1997; 95; 1930-36.

[17] Savage MP et al Acute MI in DM \& significance of Congestive Cardiac Failure as a prognostic factor Am J Cardiol 1988; 62:665-669.

[18] Mori $\mathrm{H}$ et al Abnormalities in pulmonary function in patients with NIDDM. Internal Medicine 1992; 31; 189193.

[19] Vrack R Comparison of the microvascular lesions in DM with those of normal ageing.A J Am Geriatric Soc 1982; 30; 201-205.

[20] Sandler M et al. Cross-section study of pulmonary function in patients with IDDM. Am Rev respir dis 1987; 135; 223-229.

[21] Bell et al. Are reduced lung volumes in IDDM due to defect in connective tissue. Diabetes1988; 37; 829-831.

[22] Cooper BG et al Lung functions in patients with DM Respir Med 1990; 84:235-

[23] Innocenti $F$, et al. Indications of reduced lung function in type 1 DM. Diabetes res clinical pract 1994; 25:161-168.

[24] Niranian V et al. Glycemic control \& cardiopulmonary function in patients with NIDDM Am J Med 1997:103:504-513.

[25] Sandler M et al. is the lung a "target Organ" in DM? Arch Intern med1990; 150:1385-1388. 
[26] Schernthaner G at al. Lung elasticity in juvenile DM. Am Rev Respir Dis 116; 38; 1977.

[27] Sandler $M$ et al. Pulmonary function in IDDM with limited joint mobility. Chest 90; 670.1986.

[28] Nishida O. et al Pulmonary functions in healthy subjects $\&$ its prediction spirography in adults $\mathrm{J}$ clin pathol 24:833, 1976.

[29] Hara $\mathrm{H}$ et al Pulse wave velocity (PWV) in diabetes \& Japanese-Americans in Hawai J japn Diab SOC 29: 737, 1986.

[30] Arita K et al Effects of smoking on pulmonary function J Hiroshima Med Asso 30; 1058, 1977.

[31] Sandler et al Cross-section study of pulmonary function in patients with IDDM Am Rev Respir Dis 135; 223, 1987.
[32] Sugawara K et al. Diffusion Capacity of the lung \& low $\mathrm{PaO} 2$ in DM J Anaes 28:1722.1979.

[33] Schenthaner $G$ et al. Lung elasticity in juvenile-onset DM., Am Rev Respire Dis, 1977, 113,37-41.

[34] Schernthaner G. et al. Lung elasticity in juvenile onset DM Am Rev Respire Dis 1977, 116, 544-546.

[35] Oulhen $\mathrm{Ph}$, et al. Contribution a I "etude de la function respiratoire du NIDDM Contribution a I "etude de la function respiratoire du NIDDM Rev Mal Respire 1982, $10,213-224$.

[36] Jensen G et al. Epidemiology of chest pain \& angina pectoris (theses) Acta Med Scand 1984, Suppl.682. 